\title{
Efficient XML Data Dissemination with Piggybacking
}

\author{
Chee-Yong Chan \\ National University of Singapore \\ chancy@comp.nus.edu.sg
}

\author{
Yuan $\mathrm{Ni}$ \\ National University of Singapore \\ niyuan@comp.nus.edu.sg
}

\begin{abstract}
Content-based dissemination of XML data using the publishsubscribe paradigm is an effective means to deliver relevant data to interested data consumers. To meet the performance challenges of content-based filtering and routing, two key optimizations have been developed: the use of efficient indexes to speed up subscription filtering, and the use of effective aggregation algorithms to reduce the number of subscriptions. The effectiveness of both these techniques are, however, limited to locally improving the performance of individual routers. In this paper, we propose a novel and holistic optimization approach that allows a downstream router to leverage the subscription matchings done by upstream routers to reduce its own filtering work. This is achieved by piggybacking useful annotations to the XML document being forwarded. We explore several design options and tradeoffs of this novel optimization approach. Our experimental results demonstrate that our piggyback optimization achieves significant performance improvement under various conditions.
\end{abstract}

Categories and Subject Descriptors: H.2.4 [Information Systems]: Systems - Query processing

General Terms: Algorithms, Design, Performance

Keywords: annotation, data dissemination, piggybacking, pub/sub system, XML, XPath

\section{INTRODUCTION}

The ubiquity of XML data and the effectiveness of the content-based pub/sub-based paradigm [23] of delivering relevant information has led to a lot of interest in content-based dissemination of XML data (e.g., $[8,12,11])$. In the pub/sub environment, an overlay network of application-level routers (or message brokers) is used to asynchronously forward documents generated by data publishers to relevant data subscribers (or consumers) based on matching the document contents against the consumers' subscriptions. Each subscriber first needs to pre-register a subscription to its local

Permission to make digital or hard copies of all or part of this work for personal or classroom use is granted without fee provided that copies are not made or distributed for profit or commercial advantage and that copies bear this notice and the full citation on the first page. To copy otherwise, to republish, to post on servers or to redistribute to lists, requires prior specific permission and/or a fee.

SIGMOD'07, June 12-14, 2007, Beijing, China.

Copyright 2007 ACM 978-1-59593-686-8/07/0006 ...\$5.00. router, specifying the type of data that he is interested in receiving. A routing protocol (e.g., [13]) is used to set up a routing table at each router to record both its local subscriptions as well as the subscriptions from its neighboring routers. For each document received by a router, the router compares the document contents against the subscriptions in its routing table and forwards the document to the matching local subscribers and neighboring routers.

Content-based data dissemination has traditionally been examined in the context of simple subscription specifications, such as matching of keywords or conjunction of basic comparison predicates on attribute values (e.g., [7, 24, 10]). The recent interest in using the more expressive XPathbased subscriptions for effective dissemination of XML documents has increased the complexity of the content-based subscription matching problem. Thus, there is a even greater need for effective optimization techniques to meet the performance challenges of content-based dissemination of XML data. The existing research efforts have focused on two key optimizations to minimize the number of subscription matchings. The first optimization, which has attracted the most attention, is to exploit efficient index structures (e.g., $[12,14,8,9,16,17,19,21,22,26])$ to perform selective matching with only a small subset of potentially matching subscriptions. The second optimization uses aggregation algorithms to summarize an initial set of subscriptions into a smaller set of generalized subscriptions (based on subscription containment properties) to reduce the number of subscriptions and the matching overhead $[11,26]$.

The effectiveness of the existing optimizations are, however, limited to only locally improving the performance of the individual routers. Specifically, the fact that routers are interconnected and related (in terms of the containment relationships of their subscriptions) is not being fully exploited to optimize the subscription matching.

To appreciate the new optimization opportunities and motivate our approach, consider how a document $D$ is being routed from an upstream router $R_{i}$ to a downstream router $R_{j}$ in a typical pub/sub system. On receiving $D, R_{i}$ parses and processes $D$ against the set of subscriptions $S_{i}$ stored in its routing table. Once a matching subscription $s \in S_{i}$ (that is associated with $R_{j}$ ) is detected, $R_{i}$ then forwards $D$ to $R_{j}$. A similar processing of $D$ is then repeated at $R_{j}$ but with the matching now being done against a different set of subscriptions $S_{j}$ in $R_{j}$ 's routing table. Our new optimization idea is motivated by the following two observations on the matching and routing process. Firstly, the overall processing being done at the different routers during the dissemination 
of a document can be viewed as essentially processing the same data (i.e., XML document) against a sequence of collections of queries (i.e., sets of subscriptions along each path of forwarding routers). Secondly, the sequence of collections of queries being processed are not independent as they are partially related by a "containment property" that determines whether or not a document is to be forwarded to a downstream router. Specifically, the sets of subscriptions $S_{i}$ and $S_{j}$ are related in that the subscriptions $S_{j}$ in the downstream router are being aggregated (or summarized) into a smaller set of subscriptions $S_{j}^{\prime}$ that is stored in the upstream router $R_{i}$ 's routing table (i.e., $S_{j}^{\prime} \subseteq S_{i}$ ) such that if a document $D$ does not match any of the subscriptions in $S_{j}^{\prime}$, then $D$ will certainly not match any of the subscriptions in $S_{j}$ (i.e., $S_{j}^{\prime}$ is "contained by" by $S_{j}$ ). Consequently, $R_{i}$ needs to forward $D$ to $R_{j}$ only if $D$ matches some subscription in $S_{j}^{\prime}$.

Thus, given that the same document $D$ is being processed against related sets of subscriptions, each upstream router $R_{i}$ can help to optimize the performance of its downstream router $R_{j}$ (and thereby reduce the overall processing time to deliver $D$ to relevant subscribers) by passing along some useful information to $R_{j}$ (about $D$ as well as the about related queries that $R_{i}$ has processed) when it forwards $D$ to $R_{j}$. $R_{j}$ can then try to exploit the hints that it receives from $R_{i}$ to optimize its own processing of $D$. There are two key ways that a downstream router $R_{j}$ can optimize its processing and matching of $D$ by exploiting additional hint information from its upstream router $R_{i}$ :

1. The hint could enable $R_{j}$ to quickly determine that $D$ be forwarded to a downstream router $R_{k}$ without requiring $R_{j}$ to parse and process $D$.

2 . The hint could enable $R_{j}$ to quickly detect that a portion $S_{j}^{\prime} \subseteq S_{j}$ of the subscriptions in $R_{j}$ 's routing table are guaranteed not to match $D$, and $R_{j}$ can therefore speed up its matching of $D$ against the smaller set $\left(S_{j}-S_{j}^{\prime}\right)$ instead of $S_{j}$.

We illustrate the above optimization opportunities with the following two examples.

Example 1.1 Consider the following routing of a document $D$ among three routers $\left(R_{i}, R_{j}\right.$, and $\left.R_{k}\right)$, where $D$ is first forwarded from $R_{i}$ to $R_{j}$ due to a matching subscription $/ a / / d \in S_{i}$ (that is associated with $R_{j}$ ); and then $D$ is then forwarded from $R_{j}$ to $R_{k}$ due to a matching subscription $/ a / b / c / d \in S_{j}$ (that is associated with $R_{k}$ ). Observe that if $R_{i}$ had forwarded to $R_{j}$ (along with $D$ ) the additional information on the data bindings for the matching subscription $/ a / / d \in S_{i}$; i.e., that the wildcard "//" in $/ a / / d$ actually matches the data path "b/c" in $D$, then $R_{j}$ could have very efficiently determined that $D$ matches the subscription $/ a / b / c / d \in S_{j}$ without actually having to parse and process $D$ against the subscriptions in $S_{j}$. In this way, $R_{j}$ is able to speed up the forwarding of $D$ to $R_{k}$ and thereby reduce the overall processing time to disseminate $D$ to relevant subscribers.

Example 1.2 Consider the scenario where a router $R_{i}$ needs to forward a document $D$ to its downstream router $R_{j}$, and that after having parsed and processed $D$ against $S_{i}, R_{i}$ has obtained the following information about $D$ and $S_{i}$ : (H1) $D$ does not match some subscription $s \in S_{i}$ that is associated with $R_{j}$; (H2) the data pattern "x/y/z" occurs in $D$ with its last occurrence located at some position $p$ within $D$; and (H3) the data pattern "a/b/c" does not occur at all in $D$. Observe that each of these three pieces of information could be forwarded to $R_{j}$ as hints to optimize the performance of $R_{j}$. For (H1), $R_{j}$ can use the non-matching subscription $s \in S_{i}$ to identify the subset of subscriptions $S_{j}^{\prime} \subseteq S_{j}$ in $R_{j}$ that were aggregated to $s$ (i.e., subscriptions that are guaranteed to not match $D$ ), and exclude matching $D$ against such subscriptions to improve the matching performance. For (H2), once $R_{j}$ has parsed $D$ beyond position $p, R_{j}$ can conclude that there will not be any new matches of subscriptions that contain the data pattern $x / y / z$, and therefore such subscriptions can be excluded from further matching and processing. Finally, (H3) can be treated as a special case of (H2) with $p$ being at the starting position of the document $D$. Thus, $R_{j}$ can ignore matching $D$ against the subscriptions in $S_{j}$ that contain the data pattern $a / b / c /$ right at the beginning of $D$.

In this paper, we propose an orthogonal and holistic optimization that enables a downstream router to leverage the subscription matching work completed by upstream routers to optimize its own performance. To facilitate such "collaborative" processing, a router is allowed to piggyback some additional useful information (that is derived from its subscription processing) as header annotations to an XML document before forwarding it to a downstream router. On receiving an annotated document, a router first pre-processes the header annotations to check if the hints specified in the annotations could enable any immediate forwarding decisions (without processing the document) or if they could be used to reduce the effective number of subscriptions in its router table that need to be matched against the document. We refer to this approach to optimize performance as piggyback optimization.

Note that this new optimization could be used in combination with the existing optimizations proposed for pub/sub systems (i.e., subscription indexing and aggregation).

There are three key design issues to be addressed for our piggyback optimization approach:

1. What type of information is useful to piggyback?

2. How can such information be efficiently computed by a forwarding router and exploited by a receiving router?

3. How does this optimization impact the data matching protocol (i.e., when a router $R_{i}$ detects that some subscription corresponding to a downstream router $R_{j}$ matches a document $D$, should $R_{i}$ forward $D$ immediately to $R_{j}$ ? And should $R_{i}$ continue matching $D$ against other subscriptions related to $R_{j}$ ?)

As there are many possible types of hints that could be forwarded along with a document, forwarding too much hints could increase both the transmission cost as well as the overhead of pre-processing the hints and thereby possibly negating the potential performance improvements. Thus, the hints need to be selected judiciously to balance these tradeoffs. Intuitively, a hint is preferred if it is more likely to be beneficial and can be efficiently computed by the upstream router and exploited by the downstream router.

In this paper, we examine and evaluate several design options for piggyback optimization. Our experimental study demonstrates that our proposed piggyback optimization is 


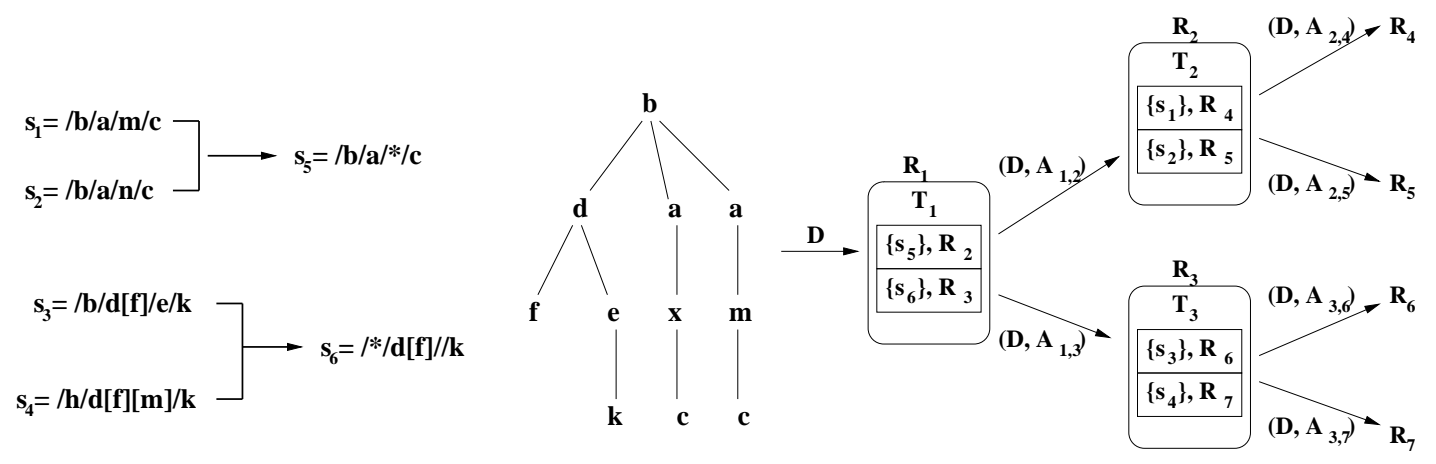

(a) Aggregating and aggregated subscriptions

(b) XML document, D

(c) Routing tables

Figure 1: XPath Subscriptions, XML Document, and Routing Tables

indeed a feasible and effective technique to improve the performance of content-based dissemination of XML data. In particular, our results show that the overhead of creating and preprocessing annotations is low relative to the performance improvement obtained from minimizing the number of subscription matchings using the annotated information. Our results indicate that the piggyback optimization is effective to improve the performance at various conditions, especially when the matching occurs at the late part of the document, the performance is improved by a factor of 2 .

Contributions. The key contributions of this paper include: (1) the proposal of a novel, holistic optimization technique for XML data dissemination called piggyback optimization that enables upstream routers to pass useful hints (in the form of document header annotations) to optimize the performance of downstream routers. This new optimization is orthogonal to the existing indexing and subscription aggregation optimizations; (2) a thorough study of the design options and issues for piggyback optimization; and (3) a comprehensive experimental performance evaluation demonstrating the efficiency of piggyback optimization.

Organization. The rest of this paper is organized as follows. Section 2 presents further background and introduces terminologies and notations. Section 3 presents our piggyback optimization technique. We discuss the mechanism to generate the annotations in Section 4 and the approach to process the annotated document in Section 5. Section 6 presents experimental results, and related work is discussed in Section 7. We conclude our paper in Section 8.

\section{BACKGROUND AND NOTATIONS}

In this section, we provide some further background on content-based XML dissemination and introduce some related terminologies and notations.

Pub/Sub systems. In a pub/sub environment, each data consumer registers his subscription to his local router. In order for a router to know about subscriptions that have been registered with other routers, a routing protocol is used by the routers in the overlay network to exchange subscription information so that their subscription tables are set up correctly to establish routing paths for forwarding documents.

We use $R_{i}$ to denote a router, and $T_{i}$ to denote the set of subscription entries in its routing table (refer to Fig. 1(c)).
Conceptually, each entry in $T_{i}$ is of the form $\left(S_{j}, p_{j}\right)$, where $S_{j}$ denotes a set of subscriptions and $p_{j}$ denotes a unique identifier that refers to either a local subscriber of $R_{i}$ or a neighboring router of $R_{i}$. For a given document $D$, we use $S_{j}^{+}(D)$ and $S_{j}^{-}(D)$ to denote, respectively, the subset of subscriptions in $S_{j}$ that matched and did not match $D$ (i.e., $\left.S_{j}=S_{j}^{+}(D) \cup S_{j}^{-}(D)\right)$. For each incoming document $D$ to $R_{i}, R_{i}$ will forward $D$ to $p_{j}$ if and only if $S_{j}^{+}(D)$ is non-empty.

If a router $R_{i}$ forwards some document to a neighboring router $R_{j}$, we call $R_{i}$ an upstream router and $R_{j}$ a downstream router. In order for any document to be forwarded from an upstream router $R_{i}$ to a downstream router $R_{j}, R_{j}$ needs to have advertised (via some routing policy) its collection of subscriptions (i.e., $\left.U_{j}=\bigcup_{(S, p) \in T_{j}} S\right)$ to $R_{i}$ so that an entry $\left(U_{j}, R_{j}\right)$ can be recorded in $T_{i}$.

XPath subscriptions. Subscriptions on XML data are typically specified using the expressive XPath-based patterns [25]. In this paper, we focus on a commonly used and expressive fragment of XPath that uses only the child ("/") and descendant ("//") axes. The node test for each XPath step can be either an element name or a wildcard "*". Nested XPath expressions are also allowed as predicates. Fig. 1(a) gives some sample XPath expressions.

Subscription aggregation. Since the entire collection of subscriptions in $R_{i}$ (i.e., $U_{i}=\bigcup_{(S, p) \in T_{i}} S$ ) is generally large, $R_{i}$ needs to summarize (or aggregate) $U_{i}$ to a smaller set $\mathcal{S}_{i}^{\prime}$ of aggregated subscriptions before advertising it to its neighboring routers. To preserve forwarding correctness, $\mathcal{S}_{i}^{\prime}$ needs to satisfy the following containment property w.r.t. $U_{i}$ : for every document $D$, if $D$ matches some subscription $s \in U_{i}$, then there must exist some subscription $s^{\prime} \in \mathcal{S}_{i}^{\prime}$ such that $D$ also matches $s^{\prime}$. We say that $\mathcal{S}_{i}^{\prime}$ contains $U_{i}$ (or $U_{i}$ is contained by $\mathcal{S}_{i}^{\prime}$ ), denoted by $U_{i} \sqsubseteq \mathcal{S}_{i}^{\prime}$. Similarly, we say that a subscription $s^{\prime}$ contains another subscription $s$, denoted by $s \sqsubseteq s^{\prime}$, if $\{s\} \sqsubseteq\left\{s^{\prime}\right\}$. The importance of the containment property (i.e., $U_{i} \sqsubseteq \mathcal{S}_{i}^{\prime}$ ) is that using $\mathcal{S}_{i}^{\prime}$ in place of $U_{i}$ for document matching will guarantee that there are no false negatives (i.e., documents not being forwarded when they should); however, false positives can arise (i.e., documents being forwarded when they need not) which are tolerable and do not compromise correctness.

Several algorithms (e.g., $[11,26])$ have been developed to 
aggregate a set of subscriptions $S$ into a smaller set $S^{\prime}$ such that $S \sqsubseteq S^{\prime}$, and they are all formulated (at a high level) in terms of the following two steps: first, partition $S$ into a collection of disjoint subsets $S_{1}, \cdots, S_{m}$, where $m<|S|$; next, aggregate each $S_{i}$ into a single subscription $s_{i}^{\prime}$ (i.e., $\left.S_{i} \sqsubseteq\left\{s_{i}^{\prime}\right\}\right)$ to obtain $S^{\prime}=\left\{s_{1}^{\prime}, \cdots, s_{m}^{\prime}\right\}$ with the properties that $S \sqsubseteq S^{\prime}$ and $\left|S^{\prime}\right|<|S|$. In addition, to ensure that the aggregated subscriptions are space-efficient, a space bound is generally impose on $S^{\prime}$ to limit the total number of query steps among all the queries in $S^{\prime}$.

For each of the subscriptions $s \in S_{i}, S_{i} \sqsubseteq S^{\prime}$, that becomes aggregated to $s_{i}^{\prime} \in S^{\prime}$ (i.e., $s \sqsubseteq s_{i}^{\prime}$ ), we refer to $s$ as an aggregating subscription, and refer to $s_{i}^{\prime}$ as an aggregated subscription of $s$. A subscription $s^{\prime}$ is said to be a simple aggregated subscription of another subscription $s$ (or $s$ is a simple aggregating subscription of $s^{\prime}$ ) if (1) $s \sqsubseteq s^{\prime}$, and (2) $s^{\prime}$ can be made to match $s$ by simply substituting each wildcard (i.e., ${ }^{*}$ and $/ /$ ) in $s^{\prime}$ with some path of data element names.

Example 2.1 Consider the set of XPath expressions $S=$ $\left\{s_{1}, s_{2}, s_{3}, s_{4}\right\}$ in Fig. 1(a). One way to aggregate $S$ into a smaller set is to first partition $S$ into two subsets $S_{1}=$ $\left\{s_{1}, s_{2}\right\}$ and $S_{2}=\left\{s_{3}, s_{4}\right\}$; followed by aggregating $S_{1}$ and $S_{2}$, respectively, into $s_{5}$ and $s_{6}$ as shown in Fig. 1(a). It can be verified that $S_{1} \sqsubseteq\left\{s_{5}\right\}$ and $S_{2} \sqsubseteq\left\{s_{6}\right\}$. We say that $s_{5}$ and $s_{6}$ are, respectively, the aggregated subscriptions of $S_{1}$ and $S_{2}$; and the subscriptions in $S_{1}$ and $S_{2}$ are aggregating subscriptions. Note that $s_{6}$ is a simple aggregated subscription of $s_{3}$ (by substituting the ${ }^{*}$ and $/ /$ in $s_{6}$, respectively, with elements $\mathrm{b}$ and e), but $s_{6}$ is not a simple aggregated subscription of $s_{4}$ (since no substitution in $s_{6}$ can create the path $d / m$ in $\left.s_{4}\right)$.

\section{PIGGYBACK OPTIMIZATION}

In this section, we present a novel approach to optimize the subscription matchings at a router. Our technique, which we termed piggyback optimization, is orthogonal to the two existing optimizations, namely, indexing techniques and subscription aggregation algorithms, that are also targeted at improving the routers' performance.

As motivated in the introduction, the central idea behind piggyback optimization is to optimize the performance of a router by leveraging information from the work done by its upstream router. This is possible because both the upstream and downstream routers are processing the same document against subscriptions that are partially related (due to subscription containment relationships). Thus, an upstream router could pass to its downstream router some useful hints (along with the document being forwarded) about properties of the document and/or matching/non-matching subscriptions that it has encountered to enable the downstream router to optimize its performance by expediting the forwarding of the document (without processing the document) and/or speeding up its subscription matching process.

In our proposed piggyback optimization, the hints from an upstream router are disseminated to its downstream router in the form of header annotations in the document. On receiving an annotated document, a router will first preprocess the header annotations to optimize the subsequent processing of the document. There are three key design issues that need to be addressed for our piggyback optimization approach: (1) What type of information is useful

\begin{tabular}{|c||l|l|}
\hline \multicolumn{1}{|c||}{ Subscription } & \multicolumn{1}{|c|}{ Data } \\
\hline \hline Positive & $\begin{array}{l}\text { Data bindings for } \\
\text { matching subscrip- } \\
\text { tions }\end{array}$ & $\begin{array}{l}\text { Positions of last occur- } \\
\text { rences of data patterns }\end{array}$ \\
\hline Negative & $\begin{array}{l}\text { Non-matching sub- } \\
\text { scriptions }\end{array}$ & $\begin{array}{l}\text { Non-occurring data } \\
\text { patterns }\end{array}$ \\
\hline
\end{tabular}

Figure 2: Types of Annotations

to piggyback? (2) How can such information be efficiently computed by an upstream router and exploited by its downstream router? (3) How does this optimization impact the matching protocol?

In this paper, we use $A_{i, j}$ to denote the header annotations that an upstream router $R_{i}$ adds to a document $D$ before forwarding it to a downstream router $R_{j}$. The annotated document that $R_{j}$ receives from $R_{i}$ is denoted by (D, $A_{i, j}$ ).

\subsection{Types of annotations}

The first key issue for the piggyback optimization technique is to decide on what types of information to include in the header annotation of a document to optimize performance. Our design of the annotated information is guided by three performance-related requirements. Firstly, it should be concise so that it incurs minimal processing overhead in terms of parsing and transmitting the additional header information. Secondly, it should be efficiently generated so that the computation overhead incurred by the upstream router does not offset any performance gains of its downstream routers. Thirdly, it should be effective in that a downstream router can efficiently preprocess the annotations to optimize its subscription matching performance.

Let us consider the possible sources of useful information that an upstream router $R_{i}$ can pass on to a downstream router $R_{j}$ along with a document $D$ that needs to be forwarded to $R_{j}$.

After having matched its own subscriptions against $D, R_{i}$ has acquired additional information about $D$ and how its subscriptions are related to $D$. We can classify this knowledge into positive and negative information:

- Positive information refers to information about (a) subscriptions in $T_{i}$ that matched $D$, and (b) patterns / properties that occur in $D$.

- Negative information refers to information about (a) subscriptions in $T_{i}$ that did not match $D$, and (b) patterns/properties that did not occur in $D$.

We shall refer to the annotations of these two types of information as positive annotations and negative annotations.

In the following, we identify two types of positive annotations (PS and PD) and two types of negative annotations (NS and ND), which are classified in Figure 2. The emphasis of the discussion in this section is on the ideas; we address the implementation issues in Section 4.

\subsubsection{Positive annotations}

A positive annotation specifies information related to either (1) a matching subscription or (2) a data pattern that occurs in the document. Subscription-related information could be used to expedite a document forwarding decision without having to process the document itself, while datarelated information could be used to reduce the effective number of subscriptions that need to be matched. 
Positive subscription (PS). A PS annotation is of the form $\left(s_{x}, B_{x}\right)$, where $s_{x}$ is some subscription detected by $R_{i}$ to match $D$ (i.e., $\left.s_{x} \in S_{j},\left(S_{j}, R_{j}\right) \in T_{i}\right)$, such that $s_{x}$ is a simple aggregated subscription of some subscription in $T_{j}$; and $B_{x}$ is the set of pairs $(l, p)$ such that $l$ specifies the position of a wildcard (i.e., $*$ and $/ /$ ) in $s_{x}$ and the $p$ is the binding of that wildcard corresponding to the detected matching. Thus, a PS annotation essentially specifies a data pattern in $D$ that matches some subscription in $T_{i}$. Such an annotation can benefit $R_{j}$ if the specified data pattern also matches some subscription $s_{y}$ in $T_{j}$ that aggregates to $s_{x}$. When this happens, $R_{j}$ can very quickly detect that $D$ matches $s_{y}$ (from processing $A_{i, j}$ ) without having to actually process $D$ (which is more costly to process than $A_{i, j}$ ). $R_{j}$ can then immediately forward $D$ to the relevant downstream router.

Example 3.1 Continuing with Example 2.1, consider the processing of the document $D$ in Fig. $1(\mathrm{~b})$ by the router $R_{1}$, which has two immediate downstream routers $R_{2}$ and $R_{3}$. $R_{1}$ first detects that $D$ matches the subscription $s_{6}$ with the wildcard $*$ and $/ /$ in $s_{6}$ matching data elements $b$ and $e$ in $D$, respectively. $R_{1}$ then adds the PS annotation $\left(s_{6},\{(1, b),(2, e)\}\right)$ into $A_{1,3}$ and forwards the annotated document $\left(\mathrm{D}, A_{1,3}\right)$ to $R_{3}$. Next, $R_{1}$ detects that $D$ also matches the subscription $s_{5}$ with the ${ }^{*}$ in $s_{5}$ matching the data element $x . \quad R_{1}$ then adds the PS annotation $\left(s_{5},\{(1, x)\}\right)$ into $A_{1,2}$ and forwards $\left(\mathrm{D}, A_{1,2}\right)$ to $R_{2}$. On receiving ( $\left.\mathrm{D}, A_{1,3}\right), R_{3}$ will first check whether any of the aggregating subscriptions for $s_{6}$ (i.e., $s_{3}$ and $s_{4}$ ) matches $s_{6}$ with the bindings $\{(1, b),(2, e)\}$. In this case, there is indeed a matching for the subscription $s_{3}$. Thus, $R_{3}$ can very quickly forward $D$ to the router $R_{6}$ without having to scan and process $D$. On the hand, when $R_{2}$ receives ( $\mathrm{D}, A_{1,2}$ ), none of the subscriptions in $R_{2}$ (i.e., $s_{1}$ and $s_{2}$ ) matches the PS $\left(s_{5},\{(1, x)\}\right)$ in (D, $\left.A_{1,2}\right)$. In this case, $R_{2}$ needs to scan and process $D$ before detecting that $s_{1}$ matches $D$. Note that if $R_{1}$ had created a PS for the second matching of $s_{5}$ in $D$ as well, $R_{2}$ would have been able to detect the matching of $s_{1}$ earlier without having to process $D$.

Positive data (PD). The purpose of a PD annotation is to specify some useful property about the data $D$ that can potentially be exploited by a downstream router $R_{j}$ to skip the matching of some of its subscriptions in $T_{j}$ thereby reducing $R_{j}$ 's processing overhead.

In this paper, we use a simple PD of the form $(p, l)$, where $p=e_{1} / e_{2} / \cdots / e_{m}$ refers to a path of element names that exists in $D$, and $l$ refers to the position of the last occurrence of $p$ in $D$. Here, the position information $l$ means that the end-tag of the element $e_{1}$ in the last occurrence of $p$ is $l^{t h}$ end-tag in $D$. To see how a $\operatorname{PD}(p, l)$ can be exploited, suppose $R_{i}$ has just completed parsing the subtree of data elements rooted at the $l^{t h}$ element in $D$, then $R_{i}$ can safely ignore all of the subscriptions that contain the pattern $p$ from further processing since such subscriptions are guaranteed not to match the remaining yet-to-be-processed portion of $D$. This subscription pruning optimization can improve performance particularly if the location $l$ is early or if there are many subscriptions in $T_{i}$ that contain $p$.

\subsubsection{Negative annotations}

The main idea behind negative annotations is to identify the set of subscriptions in the downstream router that are guaranteed not to match the document $D$ being forwarded. In this way, the downstream router can optimize its performance by eliminating the need to compare against such subscriptions against $D$.

Negative subscription (NS). The NS annotation for a downstream router $R_{j}$ (w.r.t. $D$ ) is a list of the identities of all the non-matching subscriptions in $S_{j}$ (i.e., $S_{j}^{-}(D)$ ). This information can be exploited by $R_{j}$ to skip the matching of all the aggregating subscriptions that were aggregated to $S_{j}^{-}(D)$. Specifically, for each subscription $s$ in $T_{j}$, if $s$ is an aggregating subscription of an aggregated subscription $s^{\prime} \in S_{j}^{-}(D)$ (i.e., $s \sqsubseteq s^{\prime}$ ), then by the containment property, the fact that $D$ did not match $s^{\prime}$ at $R_{i}$ necessarily implies that $D$ will not match $s$ at $R_{j}$. Thus, the matching of $s$ against $D$ at $R_{j}$ is redundant and can be skipped without affecting correctness.

Negative data (ND). Besides using non-matching aggregated subscriptions to skip the matching of corresponding aggregating subscriptions, a more general approach to enable a similar optimization is to exploit the absence of certain data patterns in $D$ to skip the matching of all subscriptions in $R_{j}$ that contain such patterns. As an example, if $R_{i}$ knows that $D$ does not contain the path of elements $p=A / B$, this negative information can be beneficial to $R_{j}$ if there is a large collection $C_{p}$ of subscriptions in $T_{j}$ that contain such a pattern $p$. By similar reasoning using the containment property, $R_{j}$ can safely skip the matching of the subscriptions in $C_{p}$. In this paper, we use simple data patterns in the form of linear paths of element names for ND annotations.

\subsection{Impact on Matching Protocol}

The matching protocol of a router $R_{i}$ refers to the two key decisions that $R_{i}$ makes when it detects that some subscription corresponding to a downstream router $R_{j}$ matches $D$. The first deals with whether $R_{i}$ should forward $D$ immediately to $R_{j}$; and the second deals with whether $R_{i}$ should continue matching $D$ against other subscriptions related to $R_{j}$.

In this section, we discuss the impact of piggyback optimization on the options for the matching protocol.

\subsubsection{Eager forwarding with skipping (ES)}

For routers in conventional pub/sub systems (without piggyback optimization), the matching protocol adopted is that when a document $D$ is detected at an upstream router $R_{i}$ to match some subscription associated with some downstream router $R_{j}, R_{i}$ will immediately forward $D$ to $R_{j}$ and skips the matching of subscriptions associated with $R_{j}$. Forwarding a document as soon as possible helps to improve response time, while skipping unnecessary subscription matchings helps to reduce the processing overhead. We refer to this conventional protocol as eager forwarding with skipping (denoted by $E S$ ).

\subsubsection{Lazy forwarding without skipping $(L)$}

The conventional $E S$ approach of forwarding $D$ to a downstream router $R_{j}$ as soon as a subscription matching for $R_{j}$ is detected generally occurs when $D$ has not been completely processed. The eager forwarding protocol has two implications with regards to the use of annotations. First, negative annotations cannot be included in the forwarded document; and second, only limited PS annotations (derived from the 
processed portion of $D$ ) can be used. Given that negative annotations could potentially help to skip a large number of subscriptions in $R_{j}$ and PS annotations could enable a document to be forwarded quickly without processing the document, it might actually be beneficial to delay the forwarding of $D$ to $R_{j}$ until $D$ has been completely processed at $R_{i}$. Clearly, for this "lazy" forwarding protocol to generate additional annotations for a matching downstream router $R_{j}$, it is necessary for $R_{i}$ to continue matching $D$ against the subscriptions that correspond to $R_{j}$. We refer to this protocol as lazy forwarding without skipping (denoted by $L$ ).

There is a performance tradeoff between the $E S$ and $L$ protocols. On the one hand, by forwarding $D$ immediately to a downstream router, $E S$ can help to reduce the response time of delivering a document to matching data consumers. On the other hand, by delaying the forwarding to generate both negative as well as complete PS annotations, $L$ can potentially minimize the matching cost at each downstream router by (1) using negative annotations to skip the processing of many subscriptions, and (2) using PS annotations to enable $D$ to be quickly forwarded without having to first parse $D . L$ is particularly cost-effective if at the time a subscription matching is detected at $R_{i}$, only a small proportion of $D$ has not been processed.

\subsection{Combining annotations and protocols}

Based on the preceding discussion, the design space of our piggyback optimization consists of four basic annotation types (PS, PD, NS, and ND) and two matching protocols $(E S$ and $L)$. A data dissemination strategy is formed by choosing a subset of annotation types together with a matching protocol. We use $P_{\beta}^{\alpha}$ to denote a dissemination strategy, where $P \in\{E S, L\}$ refers to the matching protocol used; $\alpha \subseteq\{+s,+d\}$ refers to the set of positive annotations used; and $\beta \subseteq\{-s,-d\}$ refers to the set of negative annotations used. Here, $+s,+d,-s$, and $-d$ denote, respectively, PS, $\mathrm{PD}, \mathrm{NS}$, and ND annotations. For conciseness, we use $+s d$ (resp., $-s d)$ to represent $\{+s,+d\}$ (resp., $\{-s,-d\}$ ); moreover, an empty set value is simply represented by a blank, and a singleton value $\{x\}$ is abbreviated to $x$. For example, the conventional dissemination strategy is denoted by $E S$; and a strategy that uses lazy forwarding with PS, PD, and ND annotations is denoted by $L_{-d}^{+s d}$.

Note that it is not meaningful to have a dissemination strategy that involves some negative annotation type together with the ES policy. This is because negative annotations cannot be added to $D$ if it is eagerly forwarded when the processing of $D$ has not completed. Therefore, in this paper, we do not consider dissemination strategies $E S_{\beta}^{\alpha}$ with $\beta \neq \emptyset$.

\section{GENERATING ANNOTATIONS}

In this section, we discuss the details of how an upstream router $R_{i}$ computes the various annotations (i.e., $A_{i, j}$ ) for a data $D$ to be forwarded to a downstream router $R_{j}$. Except for NS annotations, all the other annotations in $A_{i, j}$ can be created more effectively if they exploit knowledge of the subscriptions in the downstream router $R_{j}$. To achieve this, our approach generates PS, PD, and ND annotations in two steps, referred to as the offline step and online step. The offline step is performed only once as part of the routing protocol to set up the routing tables in the routers. Specifically, when a downstream router $R_{j}$ is advertising its aggregated subscriptions to each of its upstream routers, we also make use of this opportunity to transmit some useful information that is derived from $R_{j}$ 's subscriptions to the upstream routers. This derived information from $R_{j}$ will be stored by the upstream routers and used to create annotations in the online step for documents that are forwarded to $R_{j}$. The online step is performed by an upstream router each time it needs to forward a document to some downstream router.

In general, since there are many possible options for each annotation type, we devise a benefit metric for each annotation type to enable the effectiveness of annotations to be compared so that a reasonably small set of beneficial annotations can be judiciously selected (for inclusion in the document header) that both maximizes the performance improvement for the downstream router as well as ensures transmission efficiency.

In the following subsections, we describe, for each of the four annotation types, the benefit metric used to select annotations and how the annotations are created.

\subsection{Positive subscription (PS)}

Intuitively, a PS annotation $(s, B)$ is beneficial for a downstream router $R_{j}$ if $s$ is a simple aggregated subscription of many subscriptions in $R_{j}$ as this increases the chance that the pattern specified by $(s, B)$ matches a subscription in $R_{j}$. On the other hand, it is also desirable for the size of the binding $B$ to be small so that the annotation is space-efficient. We therefore define the benefit of using a subscription $s$ for a PS annotation in $A_{i, j}$ to be benefit $(s)=\frac{\left|S^{\prime}\right|}{\sum_{i \in w(s)} \text { size(i) }}$ where $S^{\prime}$ is the set of simple aggregating subscriptions of $s$ in $R_{j}, w(s)$ is the set of the wildcards in $s$, and size $(i)$ is size of binding values for the $i^{\text {th }}$ wildcard of $s$. Note that a subscription $s$ has no benefit if $S^{\prime}=\emptyset . R_{i}$ will select the most beneficial PS annotations based on the above metric.

PS annotations to be included in $A_{i, j}$ are computed in two steps. In the offline step, $R_{j}$ will identify a set of candidate subscriptions that can be used for PS annotations and advertise these candidates to $R_{i}$. In the online step, whenever $R_{i}$ needs to forward a document to $R_{j}, R_{i}$ will create PS annotations from a subset of these candidates by adding relevant data bindings.

More specifically, in the offline step, after $R_{j}$ has aggregated its subscriptions, $R_{j}$ derives the information $\left(s,\left|S^{\prime}\right|\right.$, $l$ ) for each simple aggregated subscription $s$ computed by $R_{j}$, where $S^{\prime}$ is the subset of subscriptions in $R_{j}$ that was aggregated to $s$ such that $s$ is a simple aggregated subscription of each subscription in $S^{\prime}$ (defined in Section 2); and $l$ is a list of wildcard positions indicating which of the wildcards $(/ /$ and $*)$ in $s$ need to be instantiated with data path bindings to match some aggregating subscription in $S^{\prime}$.

Example 4.1 Consider the example in Fig. 3, where the 6 subscriptions in $R_{j}$ are partitioned into three sets: $S_{1}=$ $\left\{s_{1}, s_{2}\right\}, S_{2}=\left\{s_{3}, s_{4}\right\}$, and $S_{3}=\left\{s_{5}, s_{6}\right\}$, which are aggregated, respectively, into $s_{1}^{\prime}, s_{2}^{\prime}$, and $s_{3}^{\prime}$. And for simple aggregated subscriptions $s_{1}^{\prime}$ and $s_{3}^{\prime}, S_{1}^{\prime}=\left\{s_{1}, s_{2}\right\}$ and $S_{3}^{\prime}=\left\{s_{5}\right\}$. The derived information generated by $R_{j}$ in the offline step for these aggregations are shown as $i_{1}, i_{2}$, and $i_{3}$ in Fig. 3. Observe that $i_{1}=\left(s_{1}^{\prime},\left|S_{1}^{\prime}\right|, l_{1}\right)$, where $\left|S_{1}^{\prime}\right|=2$ and $l_{1}=\{2\}$ indicating that only the second wildcard (i.e., *) requires a data binding; however, the first wildcard (i.e., //) does not require a data binding to transform $s$ to any of the subscriptions in $S$. On the other hand, since $s_{3}^{\prime}$ is the simple 


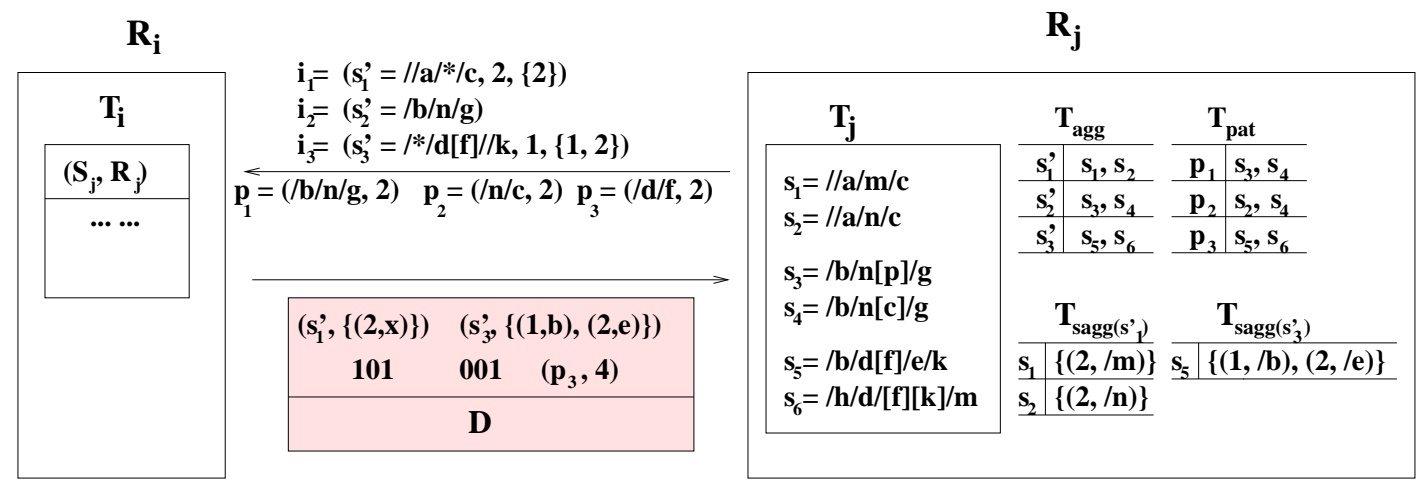

Figure 3: Generating \& Processing Annotations

aggregated subscription of only $s_{5} \in S_{3}^{\prime}, i_{3}=\left(s_{3}^{\prime},\left|S_{3}^{\prime}\right|, l_{3}\right)$, where $\left|S_{3}^{\prime}\right|=1$ and $l_{3}=\{1,2\}$.

The collection of derived information $\left(s,\left|S^{\prime}\right|, l\right)$ will be passed to upstream routers of $R_{j}$ when $R_{j}$ advertises its aggregated subscriptions to them. In the online step, when an upstream router $R_{i}$ detects a matching subscription $s$ (associated with downstream router $R_{j}$ ) while matching a document $D, R_{i}$ will first compare $s$ against the derived information from $R_{j}$ to determine whether $s$ could form a candidate PS annotation.

Example 4.2 Continuing with Example 4.1, suppose that $R_{i}$ in Fig. 3 is processing the document $D$ from Fig. 1(b). When $R_{i}$ detects that subscription $s_{1}^{\prime}$ matches the data $/ b / a / x / c$ in $D, R_{i}$ uses the derived information $i_{1}=(/ / a / *$ $/ c, 2,\{2\})$ to create the PS annotation $\left(s_{1}^{\prime},\{(2, x)\}\right)$ for $s_{1}^{\prime}$. Based on $i_{1}, R_{i}$ knows that only the second wildcard in $s_{1}^{\prime}$ requires a data binding. On further processing $D, R_{i}$ detects that subscription $s_{3}^{\prime}$ matches $D$ and creates the PS annotation $\left(s_{3}^{\prime},\{(1, b),(2, e)\}\right)$ for this matching. Fig. 3 shows the scenario where $R_{i}$ is forwarding all these PS annotations to $R_{j}$ along with $D$ (indicated by the shaded box).

\subsection{Positive data (PD)}

A PD annotation $(p, l)$ is beneficial for $R_{j}$ if $p$ is contained by many subscriptions in $T_{j}$ and the value of $l$ is small so that $R_{j}$ can skip many of its subscriptions after processing only a small portion of $D$. We therefore define the benefit of a pattern $p$ to be $\frac{\operatorname{freq}(p)}{\operatorname{pos}(p)}$, where $\operatorname{freq}(p)$ represents the number of subscriptions in $T_{j}$ that contain $p$; and $p o s(p)$ represents the position of the last occurrence of $p$ in $D . R_{i}$ then selects a subset of the PD annotations of the form $(p, p o s(p))$ that have the highest benefit values.

PD annotations are also computed in two steps. In the offline step, $R_{j}$ advertises to $R_{i}$ a small set of beneficial data patterns $P_{j}$ (together with their frequencies) derived from the subscriptions in $T_{j} . R_{j}$ selects data patterns to be included in $P_{j}$ based on the following benefit metric for a data pattern $p$ : benefit $(p)=f r e q(p) \cdot \ln (l(p)+1)$, where $\operatorname{freq}(p)$ represents the number of subscriptions in $T_{j}$ that contain $p$; and $l(p)$ represents the length of the linear pattern $p$. The function $\ln (l(p)+1)$ provides an approximate measure of the probability that the last appearance of $p$ occurs early in the document. In the online step, an upstream $R_{i}$ keeps track of $\operatorname{pos}(p)$ for each pattern $p \in P_{j}$ as it processes $D$. With the freq(.) information from $R_{j}$ and the pos(.) information that it derives, $R_{i}$ can approximately select the most beneficial PD annotations for $A_{i, j}$.

Example 4.3 Consider again the example in Fig. 3 which shows that $R_{j}$ is advertising three candidate data patterns $p_{1}, p_{2}$ and $p_{3}$ (along with their frequencies) to $R_{i}$ for possible use as PD annotations. In the online step, after $R_{i}$ has completed processing $D$ (from Fig. $1(\mathrm{~b})$ ), $R_{i}$ detects that $\operatorname{pos}\left(p_{3}\right)=4$ (note that there is no occurrence of $p_{1}$ and $p_{2}$ in $D)$. It decides to create the PD annotation $\left(p_{3}, 4\right)$ and forwards it to $R_{j}$.

\subsection{Negative subscription (NS)}

An NS annotation is a subscription $s \in T_{i}$ that did not match $D$; and it is more beneficial to $R_{j}$ if there are more subscriptions in $T_{j}$ that aggregate to $s$ as it enables $R_{j}$ to skip the processing of a larger number of subscriptions. The benefit of each $s \in T_{i}$ is therefore defined to be the number of subscriptions in $T_{j}$ that aggregates to $s$. NS annotations are computed in two steps. In the offline step, $R_{j}$ notifies to its upstream router $R_{i}$ the number of its subscriptions that aggregate to each aggregated subscription. While processing a document $D$ during the online step, $R_{i}$ selects from among the subscriptions in $T_{i}$ that did not match $D$, the subset with the highest benefit values as NS annotations. However, as the total number of subscriptions is generally not too large, all the non-matching subscriptions can be specified concisely and precisely using a bitstring with each subscription represented by a single bit such that the bit is turned on if and only if the subscription is non-matching.

\subsection{Negative data (ND)}

An ND annotation is of the form of a linear data pattern $p$ that is absent in $D$. Intuitively, a data pattern $p$ is more beneficial to $R_{j}$ if there are more subscriptions in $T_{j}$ that contain $p$ and the probability of $p$ 's occurrence in $D$ is low. In fact, ND annotations can be viewed as a special case of $\mathrm{PD}$ annotations with $\operatorname{pos}(p)=0$. Thus, we can use the same metric $f r e q(p) \cdot \ln (l(p)+1)$ (defined in Section 4.2 for PD annotations) to compare the benefit of different ND annotations. The generation of ND annotations in $A_{i, j}$ follows a similar two-step process, where $R_{i}$ advertises to $R_{j}$ a set of candidate data patterns during the offline step; and during the online step, $R_{i}$ keeps track of the candidate patterns that did not occur in the document being processed, and concisely represent the non-matching data patterns as a bitstring in the ND annotations. 
Example 4.4 After $R_{i}$ in Fig. 3 has processed the document $D$ from Fig. $1(\mathrm{~b}), R_{i}$ detects that $D$ did not match the subscription $s_{2}^{\prime}$ and that the data patterns $p_{1}$ and $p_{2}$ did not occur in $D$. Thus, $R_{i}$ can create the ND annotation 001 and the NS annotation 101 for $R_{j}$.

\section{PROCESSING ANNOTATED DOCUMENTS}

In this section, we describe the details of how a router $R_{j}$ processes an annotated document $\left(\mathrm{D}, A_{i, j}\right)$ that it receives from some upstream router $R_{i}$.

To efficiently and effectively process annotations in an annotated document ( $\left.\mathrm{D}, A_{i, j}\right)$, each downstream router $R_{j}$ maintains the following information:

Aggregation Table, $T_{a g g}$. For each aggregated subscription $s^{\prime}$ generated by $R_{j}, T_{a g g}\left(s^{\prime}\right)$ stores the set of aggregating subscriptions of $s^{\prime}$.

Simple Aggregation Table, $T_{\text {sagg }}$. For each aggregated subscription $s^{\prime}$ generated by $R_{j}$, if $s^{\prime}$ is also a simple aggregated subscription, $T_{\text {sagg }}\left(s^{\prime}\right)$ stores the set of pairs $(s, P)$, where $s$ is a simple aggregating subscription of $s^{\prime} . P$ is a set of pairs $\left(l_{i}, p_{i}\right)$, where $l_{i}$ specifies the position of a wildcard in $s^{\prime}$ and $p_{i}$ specifies a data pattern binding such that if for each $\left(l_{i}, p_{i}\right) \in P$, the $l^{\text {th }}$ wildcard in $s^{\prime}$ is replaced by the pattern $p_{i}$, then the transformed $s^{\prime}$ will match $s$.

Pattern Table, $T_{\text {pat }}$. For each data pattern $p$ that $R_{j}$ has advertised to its upstream routers during the offline step for PD or ND annotations, $T_{p a t}(p)$ stores the subset of subscriptions in $R_{j}$ that contains $p$ such that if a document $D$ does not match $p$, then $D$ also does not match any of the subscriptions in $T_{\text {pat }}(p)$.

Non-Matching Array, $A_{n o t}$. $A_{n o t}$ is a bit-array of size equal to the number of subscriptions in $R_{j}$ such that the $i^{\text {th }}$ bit corresponds to the $i^{t h}$ subscription in $R_{j}$. Each $A_{n o t}[i]$ is initialized to zero at the start when $R_{j}$ receives $D$, which could be set to one as $R_{j}$ processes the annotations and $D$. Specifically, $A_{\text {not }}[i]$ is set to one if and only if the $i^{\text {th }}$ subscription in $R_{j}$ is guaranteed not to match the document $D$ being processed. $R_{j}$ uses $A_{n o t}$ to optimize its processing of $D$ by skipping the processing of subscriptions that indicated by $A_{\text {not }}$ to be guaranteed to not match $D$.

Fig. 3 shows all the tables maintained at $R_{j}$ for the six subscriptions. Note that all the tables $T_{a g g}, T_{\text {sagg }}$ and $T_{p a t}$ are created only once in the offline step after $R_{j}$ has advertised his aggregated subscriptions and derived information to its upstream routers. These tables remained static unless there are changes to the subscriptions in $R_{j}$. The bit-array $A_{\text {not }}$ is the only structure that needs to be initialized and updated for each document that $R_{j}$ processes.

\subsection{Processing Annotations $A_{i, j}$}

We are now ready to explain how $R_{j}$ processes an annotated document $\left(\mathrm{D}, A_{i, j}\right)$ that it receives from $R_{i}$ using the following four steps.

Step 1: Processing PS Annotations. For each PS annotation $\left(s^{\prime}, B\right) \in A_{i, j}, R_{j}$ compares $\left(s^{\prime}, B\right)$ against each $(s, P) \in T_{\text {sagg }}\left(s^{\prime}\right)$. If for each pair $\left(l_{i}, p_{i}\right) \in P$, there exists a pair $\left(l_{i}^{\prime}, p_{i}^{\prime}\right) \in B$ such that $l_{i}=l_{i}^{\prime}$ and $p_{i}$ matches $p_{i}^{\prime}$, then $D$ is detected to match subscription $s$ and $R_{j}$ can immediately forward $D$ to the downstream router (say $R_{k}$ ) associated with subscription $s$ without the need to first process $D^{1}$.

\footnotetext{
${ }^{1}$ Note that the immediate forwarding due to a matching
}

Since $R_{j}$ has not yet processed $D, A_{j, k}$ needs to be derived from $A_{i, j}$; the details are described in Section 5.3.

Example 5.1 Suppose that $R_{j}$ has just received from $R_{i}$ the annotated document $\left(\mathrm{D}, A_{i, j}\right)$ (indicated by the shaded box in Fig. 3) and is processing the PS annotations. For the PS annotation $P S_{1}=\left(s_{3}^{\prime},\{(1, b),(2, e)\}\right) \in A_{i, j}$ in which $B=$ $\{(1, b),(2, e)\}, R_{j}$ will process $P S_{1}$ against each $(s, P) \in$ $T_{\text {sagg }}\left(s_{3}^{\prime}\right)$. For the tuple $s=s_{5}$ and $P=\{(1, / b),(2, / e)\}$ $\in T_{\text {sagg }}\left(s_{3}^{\prime}\right), R_{j}$ detects that it matches $P S_{1}$ since for $(1, / b)$ $\in P$ there exists a pair $(1, b) \in B$ such that $b$ matches $/ b$; and for $(2, / e) \in P$, there exists the pair $(2, e) \in B$ and $e$ matches $/ e$. Thus $R_{j}$ knows $D$ matches $s_{5}$ without processing D. $\square$

Step 2: Processing NS Annotations. For each NS annotation $s^{\prime} \in A_{i, j}, R_{j}$ knows that $D$ will not match any of the subscriptions in $T_{a g g}\left(s^{\prime}\right) . R_{j}$ therefore updates $A_{\text {not }}[i]$ to one for each aggregating subscription $s_{i} \in T_{\text {agg }}\left(s^{\prime}\right)$.

Step 3: Processing ND Annotations. For each ND annotation $p \in A_{i, j}, R_{j}$ knows that $D$ will not match any of the subscriptions in $T_{\text {pat }}(p) . R_{j}$ therefore updates $A_{n o t}[i]$ to one for each subscription $s_{i} \in T_{\text {pat }}(p)$.

Step 4: Processing PD Annotations \& D. For each $\mathrm{PD}$ annotation $(p, \ell) \in A_{i, j}, R_{j}$ will dynamically process each of them (in ascending order of their positions $\ell$ ) as part of its processing of $D$. Specifically, if $R_{j}$ has completed parsing some data element in $D$ at position $\ell$ and $(p, \ell)$ is the next-to-be-processed $\mathrm{PD}$ annotation, then $R_{j}$ knows that the data pattern $p$ will not occur in the remaining portion of $D$, and that it is redundant to process any new matchings of subscriptions in $T_{\text {pat }}(p)$. Therefore, $R_{j}$ updates $A_{n o t}[i]$ to one for each subscription $s_{i} \in T_{\text {pat }}(p)$.

\subsection{Processing Document $D$}

Based on the preceding discussion, $R_{j}$ processes all the PS, NS, and ND annotations before it begins to process $D$ (steps 1-3). In some situations (step 1), it is even possible for $R_{j}$ to forward $D$ to another downstream router without having to process $D$ at all. The PD annotations are processed (step 4) along with the normal processing of $D$.

When $R_{j}$ detects that $D$ matches some subscription $\left(S_{k}\right.$, $\left.R_{k}\right) \in T_{j}$, there are two cases to consider. If the dissemination strategy used is the $E S$ matching protocol, then $R_{j}$ will generate the appropriate annotations for $A_{j, k}$ (depending on the annotation types being used) and forward (D, $\left.A_{j, k}\right)$ to $R_{k}$. Moreover, $R_{j}$ will also skip all subscriptions associated with $R_{k}$ from further matching as it continues to process $D$; this is achieved by setting the appropriate bits in $A_{n o t}$ to one. Otherwise, if the dissemination strategy in use is the $L$ matching protocol, then $R_{j}$ will only forward $D$ to $R_{k}$ (with appropriate $A_{j, k}$ ) after it has completely processed $D$.

\subsection{Deriving Negative Annotations}

In this section, we consider the scenario where $R_{j}$ is going to forward $D$ to a downstream router $R_{k}$ without having yet completed processing the entire document $D$. An issue that arises from this situation is what are the possible negative annotations (if any) that $R_{j}$ can include in $A_{j, k}$ given that $R_{j}$ has not processed some portion of $D$. Observe that $R_{j}$ cannot arbitrarily include a subscription $s$ that has not matched the processed portion of $D$ as an NS annotation

in $A_{i, j}$ is independent of the forwarding policy being used which applies when there is a matching in $D$. 
since $s$ could potentially have matched $D$ if $R_{j}$ has processed $D$ completely.

It turns out that $R_{j}$ can actually derive some limited types of negative annotations for $A_{j, k}$ : (1)for each NS annotation $s^{\prime} \in A_{i, j}, R_{j}$ can identify the subset of subscriptions $S_{k} \subseteq T_{a g g}\left(s^{\prime}\right)$ that are associated with $R_{k}$. Thus, the subscriptions in $S_{k}$ can be included as NS annotations in $A_{j, k}$; (2)the ND annotations in $A_{i, j}$ can be directly inherited as ND annotations in $A_{j, k}$. However, since the inherited annotations are not specifically optimized for $R_{k}$ using derived information from $R_{k}$, they are generally less beneficial than the customized ND annotations.

\section{PERFORMANCE STUDY}

To verify the effectiveness of our proposed annotations in content-based dissemination of XML data, we conducted extensive experiments to compare the performance of various dissemination strategies. Our results show that the dissemination strategy $L_{-s d}^{+s}$ outperforms the conventional method $E S$ by a factor of 2 .

\subsection{Experimental Testbed}

We extended the NS2 network simulator [3] for our experiments by adding application code for content-based routing and piggyback optimization. The subscription indexing method and subscription aggregation approach implemented for each router are based on existing solutions in the literature $[12,11]$. It is important to emphasize that our proposed piggyback optimization approach is orthogonal to the specific algorithms used for filtering and aggregation.

The router network topology used was a complete binary tree with fours levels and a total of 15 routers, where each router (except for the root router) has one immediate upstream router at one level above. Data is disseminated from the root router downwards to the leaf routers. Data users can subscribe to any router; and each router aggregates all its subscriptions to a size that is $k \%$ of the original set; our experiments used values of 12.5 and 25.0 (the default value) for $k$. The network bandwidth values used were $1 \mathrm{MBps}$, 10MBps (default), and 100MBps.

Data sets. Our experiments used three synthetic data sets (1) NITF DTD [5], which has been used in previous studies $[8,14,12] ;(2)$ Treebank [6]; (3) DBLP [2]. For each data set, ten documents were generated using IBM's XML Generator [1]. In addition, we also used one real-life Protein data set [4] by extracting data from it to form small documents. The average sizes of the documents in each of the data sets are shown in Table 1, where $\mathrm{NITF}_{2}$ is the default data set. Note that the document sizes used in our experiments are typical for data dissemination settings $[14,12]$.

\begin{tabular}{cccccc}
\hline $\mathrm{NITF}_{1}$ & $\mathrm{NITF}_{2}$ & $\mathrm{NITF}_{3}$ & Treebank & DBLP & Protein \\
\hline 50 & 129.4 & 200.8 & 144.1 & 116.6 & 276081 \\
\hline
\end{tabular}

Table 1: Average document size used (\# elements)

Subscriptions. The subscriptions were generated using the XPath generator in [14], and the parameter values used are shown in Table 2 with the default values used indicated in bold.

In order to study the effect of the choice of forwarding policy on performance, it is important to be able to control

\begin{tabular}{lll}
\hline Parameter & Description & Value \\
\hline $\mathrm{L}$ & maximum number of steps & 8 \\
$\rho_{*}$ & probability of "*" & 0.1 \\
$\rho_{/ /}$ & probability of "//" & 0.1 \\
$\rho_{\lambda}$ & probability of nested paths & $0.1, \mathbf{0 . 2}, 0.4$ \\
$\theta$ & skewness of element names & $0, \mathbf{0 . 5}$ \\
$\mathrm{P}$ & \#subscriptions per node & $2500, \mathbf{5 0 0 0}, 50000$ \\
\hline
\end{tabular}

Table 2: Parameter values for subscriptions

the position in the document at which the first subscription matching is detected. Intuitively, the benefit of early forwarding is more significant if the first subscription matching occurs early in the document; while the benefit of lazy forwarding is more significant if the first subscription matching occurs late in the document. To enable the first subscription matching position to be varied, we inserted an unique element <test> in each generated document (varying the location being inserted), and also added an additional predicate //test to each generated subscription. In this way, a subscription matching can occur only after the <test $>$ element has been parsed in the document. In our experimental graphs, the first matching position is represented as a fraction $f \in[0,1]$ indicating the proportion of the document parsed before the occurrence of a first subscription matching. The values used for $f$ are $0.25,0.5,0.75$, and 1 .

Algorithms. We compared the various dissemination strategies $P_{\beta}^{\alpha}$ discussed in Section 3.3 by varying the annotation types $(\alpha$ and $\beta$ ) and matching protocols $(P)$, including the conventional approach $E S$ as a special case. The main performance metric compared is the response time, which is defined as the average time taken to disseminate a published document from its source to the relevant users. The response time for disseminating a document $D$ comprises of two key components: (1) the transmission spent in in the network, and (2) the processing time incurred by the routers to process annotations against $D$, match subscriptions against $D$, and generate annotations. Each response time reported for a data set is the average response time for disseminating the ten documents in the data set.

Our experiments were conducted on a $3 \mathrm{GHz}$ Intel Pentium IV machine with $1 \mathrm{~GB}$ main memory running Windows XP, and all algorithms are implemented in $\mathrm{C}++$.

\subsection{Experimental Results}

Effect of annotation types. Fig. 4(a) compares the performance of different annotation types using the lazy forwarding without skipping protocol as the first matching position, $f$, is being varied on the x-axis. Among the four annotation types, ND improves $L$ the most, followed by PS, NS, and PD. $L^{+d}$ (not shown in Fig. 4(a)) turns out to have similar performance as $L$, since the effectiveness of PD to skip the matching operations is limited. $L_{-d}$ performs better than $L_{-s}$ for the reason that the NITF DTD has many optional elements such that it is likely that some elements or substrings absent from the documents which makes ND more effective to eliminate non-matching subscriptions. Specifically, for our default setting, $10 \%$ of the subscriptions on each router are matching. ND can eliminate $70 \%$ of the total subscriptions while NS can eliminate only $37 \%$ of them. Our results also show that PS is more effective than NS but less effective than ND.

Observe that when both NS and ND are combined, it im- 


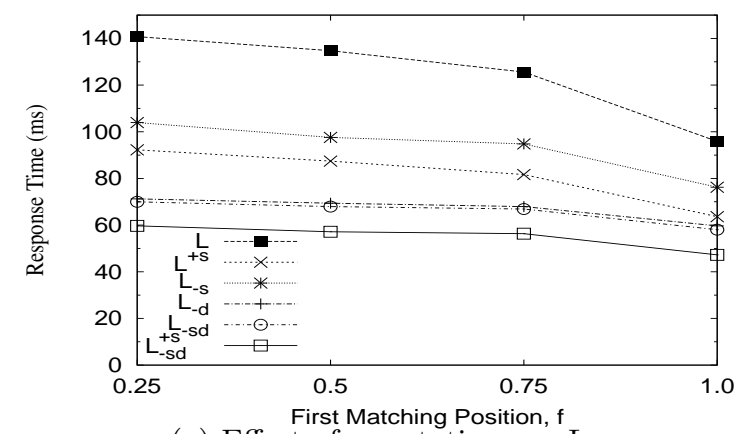

(a) Effect of annotations on $\mathrm{L}$

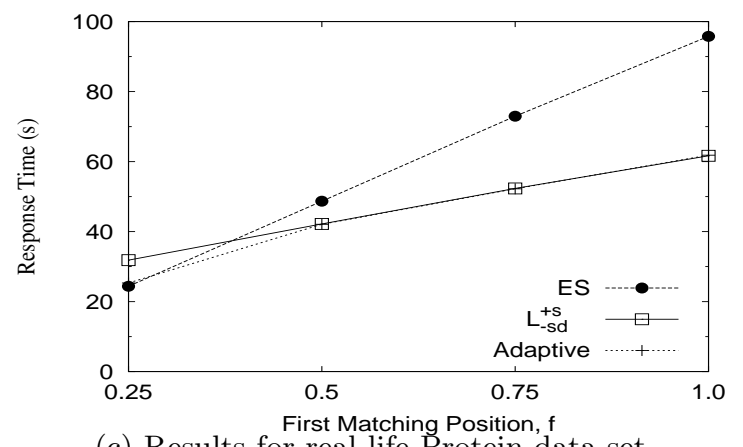

(c) Results for real-life Protein data set

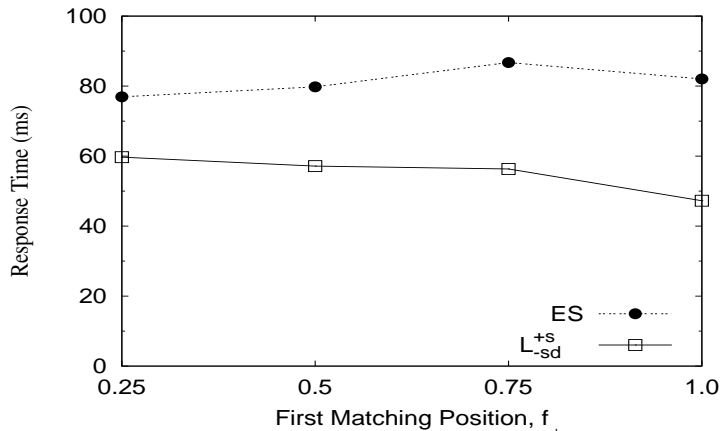

(b) Comparison of ES with $L_{-s d}^{+s}$

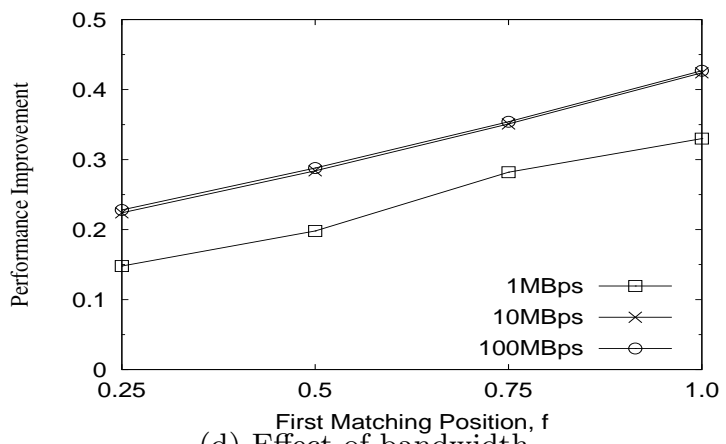

(d) Effect of bandwidth

Figure 4: Experimental results for different dissemination approaches

proves over ND only slightly. The reason is that a number of subscriptions that can be skipped using NS can also be skipped by ND; consequently, using NS in addition to ND offers very little improvement. However, as the overhead of using NS is small, adopting both negative annotations is still better than using only a single negative annotation. The performance of $L_{-s d}$ can be further optimized by also using PS, and $L_{-s d}^{+s}$ is in fact the best strategy based on lazy forwarding. This is because PS enables a document to be forwarded to some routers very quickly without parsing the document; and when this is not possible, the negative annotations are effective in skipping many subscription matchings.

On the other hand, our experimental results (not shown) indicate that positive annotations ${ }^{2}$ do not enhance the performance of the eager forwarding policy at all: $E S^{+s}$ has similar performance as $E S$, while $E S^{+d}$ actually performs worse than ES. This is because only very limited PS annotations can be used when a document is forwarded eagerly; and similar to the case for lazy forwarding, PD turns out to be not cost-effective due to the fact that PD only enables a small number of subscriptions to be skipped and its benefit is offset by its processing overhead.

Eager vs. lazy forwarding. Fig. 4(b) compares the performance of the best eager-forwarding strategy $(E S)$ and the best lazy-forwarding strategy $\left(L_{-s d}^{+s}\right)$. The results show that $L_{-s d}^{+s}$ outperforms $E S$ indicating that the slight delay incurred by lazy forwarding is compensated by the improvement gained by the downstream routers from exploiting a more complete set of annotations to optimize their processing. We also observe that as $f$ increases from 0.25 to 1 , the

\footnotetext{
${ }^{2}$ Recall from Section 3.3 that negative annotations are not meaningful for eager forwarding.
}

improvement by $L_{-s d}^{+s}$ over $E S$ also increases from $22 \%$ to $42 \%$. The reason is that as $f$ grows, the lazy forwarding in $L_{-s d}^{+s}$ incurs relatively smaller delay.

Effect of other workload. Our results with other synthetic data sets (Treebank and DBLP) show similar trends with $L_{-s d}^{+s}$ obviously outperforming $E S$, thus we omit the charts here. Fig. 4(c) shows the comparison using the reallife Protein data set. Observe that $E S$ is actually better than $L_{-s d}^{+s}$ when $f=0.25$, but as $f$ increases, $L_{-s d}^{+s}$ outperforms $E S$ with increasing margin. The reason that $E S$ performs better with $f=0.25$ is due to the large document size (about 10MB) : the benefit of the annotations is offset by the longer delay incurred by $L_{-s d}^{+s}$ when $f$ is very small. We also tried an adaptive approach where $E S$ is used when the first subscription matching occurs early and $L_{-s d}^{+s}$ is used otherwise. Our result shows that this hybrid strategy (indicated as "Adaptive" in Fig. 4(c)) outperforms ES and $L_{-s d}^{+s}$.

Effect of bandwidth. Fig. 4(d) shows the effect of the network bandwidth. With a small bandwidth of $1 \mathrm{MBps}, L_{-s d}^{+s}$ has a $15 \%$ improvement over $E S$ when $f=0.25$; which increases to over $30 \%$ when $f$ increases to 1.0 . As the bandwidth increases to $10 \mathrm{MBps}$, the performance improvement increases to $22 \%$ when $f=0.25$ and to $42 \%$ when $f=1.0$. This is because the disadvantage of $L_{-s d}^{+s}$ (in terms of the delay in forwarding) becomes even less significant (relative to the processing time incurred by the routers) with a higher network bandwidth. Not surprisingly, our results for a bandwidth of $100 \mathrm{MBps}$ are similar to the results for a bandwidth of $10 \mathrm{MBps}$. Note that, the space overhead incurred by all annotations used in $L_{-s d}^{+s}$ is 464 bytes (the size of PS, NS, and ND are 224, 160, and 80 bytes, respectively) for the default experimental setting where the size of the documents is 


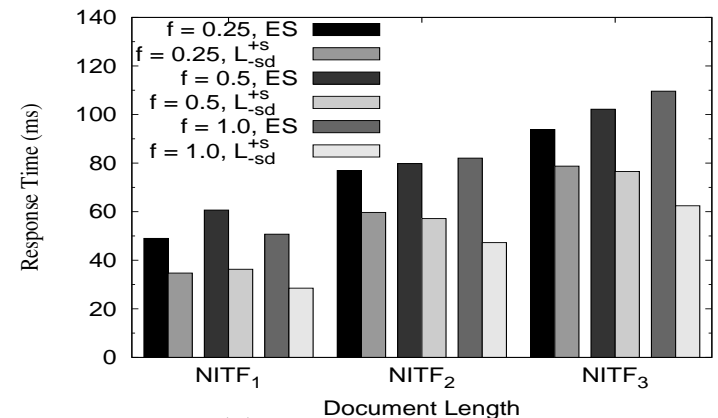

(a) Effect of data size

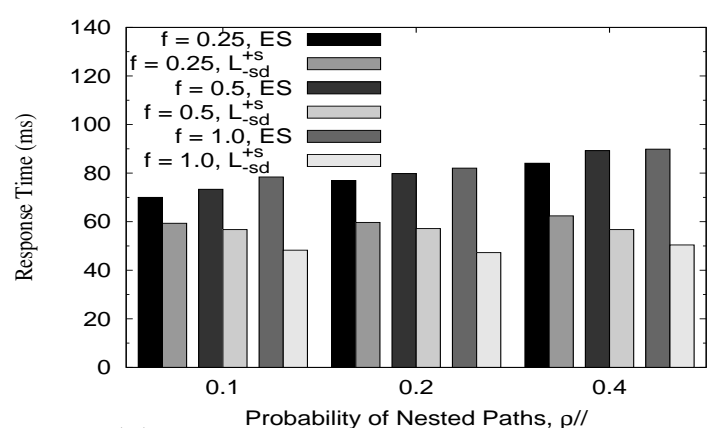

(b) Effect of subscription complexity

Figure 5: Experimental results for varying the document size and query complexity

around 7000 bytes. We can see that the size occupied by the annotations is no more than $7 \%$ of the document size. Thus, transmitting the additional annotations with the document only incurs a very small overhead in the network delay, while the speedup obtained for the processing on the routers is up to a factor of 2 .

Effect of data size. Intuitively, a larger document has two conflicting effects on the performance of $L_{-s d}^{+s}$. On the one hand, the delay incurred by $L_{-s d}^{+s}$ is expected to increase, but on the other hand, the improvement obtained from skipping subscription matchings would also become more significant with a larger document. Our experimental results shown in Fig. 5(a) (using data sets $\mathrm{NITF}_{1}, \mathrm{NITF}_{2}$ and $\mathrm{NITF}_{3}$ ) indicate that for larger documents (e.g. $\mathrm{NITF}_{3}$ ) when $f=$ 0.25 , the improvement of $L_{-s d}^{+s}$ over $E S$ becomes smaller (i.e. $16 \%$ ), compared with the improvement for $\mathrm{NITF}_{2}$ (i.e. $22 \%$ ). But their performance margin widens significantly as the value of $f$ increases. When $f=1$, the improvement of $L_{-s d}^{+s}$ over $E S$ for $\mathrm{NITF}_{3}$ is $41 \%$, which is almost the same with $\mathrm{NITF}_{2}$ (i.e. $42 \%$ ).

Effect of subscription complexity. By varying the $\rho_{\lambda}$ parameter to increase the complexity of the subscriptions, we observe that the performance gain of $L_{-s d}^{+s}$ over $E S$, shown in Fig. 5(b), becomes more significant with more complex subscriptions. In particular, when $\rho_{\lambda}$ increases from 0.1 to 0.4 , the improvement of $L_{-s d}^{+s}$ over $E S$ (with $f=1.0$ ) increases from $38 \%$ to $44 \%$. The reason is because the processing cost of the subscriptions increases with their complexity; thus, the savings from skipping subscription matchings also become more significant.

Effect of number of subscriptions. When the number of subscriptions P is increased from 2500 to 5000 , we observe that the improvement of $L_{-s d}^{+s}$ over $E S$ becomes larger (from $35 \%$ for $\mathrm{P}=2500$ to $42 \%$ for $\mathrm{P}=5000$ ). This is due to an increase in the number of subscriptions that can be skipped by NS and ND. Fig. 6 shows the results when $P$ is increased to 50000 . We observe that for larger $f$ (i.e. $f=0.75$ and $f=1.0)$, the improvement of $L_{-s d}^{+s}$ over $E S$ stays the same (e.g., $41 \%$ at $f=1.0$ ). However, for smaller values of $f$, the improvement of $L_{-s d}^{+s}$ over $E S$ diminishes slightly; e.g., with $f=0.5$, the improvements decreases from $28 \%$ for $\mathrm{P}$ $=5000$ to $11 \%$ for $\mathrm{P}=50000$. When $f=0.25$, ES is more efficient than $L_{-s d}^{+s}$. The reason is because for $L_{-s d}^{+s}$, the root router needs to match against all the subscriptions; and when $f=0.25$, the delay at the root router is increased

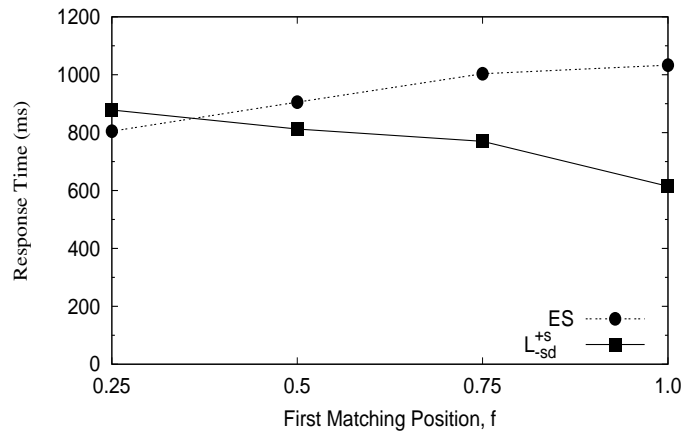

Figure 6: Effect of number of subscriptions

more significantly for $L_{-s d}^{+s}$ (relative to $E S$ ) such that the the overall efficiency of $L_{-s d}^{+s}$ becomes diminished.

Other experiments. We also vary other parameters $(k=$ $12.5, \theta=0$ ) separately with the following results :(1) With a smaller value of $k$, PS becomes slightly less effective since the more brute aggregation makes the simple aggregation requirements harder to satisfy; and the performance of NS decreases a little as well due to the less accuracy of the aggregation; (2) Our results show that varying the distribution to generate the subscriptions has little effect on the performance trend.

Throughput comparison. Although $L_{-s d}^{+s}$ performs better in terms of the response time, the results show that the throughput of $L_{-s d}^{+s}$ is slightly worse than $E S$ : when $f=0.25$, the throughput of $L_{-s d}^{+s}$ is $72 \%$ of that of $E S$; when $f=1.0$, it increases to $88 \%$ of $E S$ 's throughput. The total throughput is determined by the slowest router in the system. In $L_{-s d}^{+s}$, the root router is the bottleneck since there are no annotations that can be used and all subscriptions have to be evaluated, thus the throughput of $L_{-s d}^{+s}$ loses to $E S$. However, at all downstream routers, the disseminated documents are processed much faster using $L_{-s d}^{+s}$ than using $E S$ because of the effective annotation inserted. Therefore, if we use a more powerful router at the root node, $L_{-s d}^{+s}$ can also achieve higher throughput than ES.

\subsubsection{Discussions}

Based on our experimental results, we have the following observations on the effectiveness of the various annotation 
types. First, the effectiveness of PD annotations is found to be limited as only very few subscriptions can be pruned using PD annotations and the marginal saving from using PD annotations is offset by its overhead. Second, comparing ND and NS annotations, the former is generally more effective than the latter. This is due to a combination of two reasons. As the disseminated documents are small and the data schema has many optional elements, many of the elements in the schema (and hence also appearing in many queries) do not occur in the small documents. Thus, ND annotations can help prune many queries in a downstream router. Moreover, the process of aggregating subscriptions often results in a document $D$ matching an aggregated subscription $s^{\prime}$ in an upstream router even when all of the aggregating subscriptions of $s^{\prime}$ in a downstream router do not match $D$. Finally, the relative effectiveness between ND and PS annotations is less clear due to the different nature of their benefits. Recall that the benefit of ND annotations is in reducing subscription matching in a downstream router, while the benefit of PS annotations lies in enabling the forwarding of document without processing it. Neither ND nor PS annotations are found to be significantly more effective than the other in our experiments.

\section{RELATED WORK}

Work on optimizing the performance of content-based dissemination of XML data has mainly focused on minimizing the number of subscription matchings via two techniques: indexing methods to enable selective subscription matchings $[8,14,17,19,26,16,22,9,21]$, and subscription aggregation techniques to summarize a set of subscriptions into a smaller set of generalized subscriptions $[11,26]$. A recent interesting direction explored by Fisher and Kossmann [15] examines batched processing of multiple documents which is distinct from our work.

The recent work by Gupta, Halevy, and Suciu [18, 20] also deals with using document header annotations in contentbased data dissemination; however, the problem definition and setting are completely different. In their work, each router has a workload of simple conjunctive queries that need to be processed on each incoming document. Their motivation is to try to avoid parsing the document at each router by using a set of views whose results are pre-computed and stored as the document header annotation. Each view's result is represented by a byte offset in the XML document; and the header annotation for a document is statically determined by a centralized server that has knowledge of all the routers' workloads. Our paper is fundamentally different from theirs in the following four ways: (1) our work is motivated by using annotations to leverage the processing done by upstream routers to optimize subscription matchings at downstream routers; (2) our annotations are not static byte offsets but refer to data patterns and subscriptions which can be changed as the document is being routed; (3) our annotations are not precomputed by a centralized entity, but are instead computed dynamically by each forwarding router; and (4) our annotations exploit the containment property between aggregated and aggregating subscriptions for optimization.

\section{CONCLUSIONS}

In this paper, we have proposed a novel approach to op- timize the performance of content-based dissemination of XML data by piggybacking useful annotations to the document being forwarded so that a downstream router can leverage the processing done by its upstream router to reduce its own processing overhead. We have examined a large design space of dissemination strategies that combine four types of annotations and two forwarding policies. Our experimental study demonstrates both the feasibility and effectiveness of the new approach. In particular, the strategy of combining lazy forwarding with three types of annotations turn out to be the best option that outperforms the conventional method by a factor of 2 . As part of future work, we intend to explore the performance tradeoffs of adopting other annotation types.

\section{REFERENCES}

1] A. L. Diaz, D. Lovell (1999) XML Generator. http://www.alphaworks.ibm.com/tech/xmlgenerator.

[2] DBLP. http://www.acm.org/sigmod/dblp/db/about/dblp.dtd.

[3] NS2. http://www.isi.edu/nsnam/ns/.

[4] Protein. http://pir.georgetown.edu.

[5] R. Cover (1999) The SGML/XML web page. http:// www.oasis.open.org/cover/sgml-xml.html.

[6] Treebank. http://www.cis.upenn.edu/ treebank/.

[7] M. K. Aguilera, R. E. Strom, D. C. Sturman, M. Astley, and T. D. Chandra. Matching events in a content-based subscription system. In PODC, 1999.

[8] M. Altinel and M. Franklin. Efficient filtering of XML documents for selective dissemination of information. In VLDB, 2000.

[9] N. Bruno, L. Gravano, N. Koudas, and D.Srivastava. Navigation- vs. index-based XML multi-query processing. In ICDE, 2003.

[10] A. Carzaniga, D. Rosenblum, and A. Wolf. Design and evaluation of a wide-area event notification service. ACM TOCS, 19(3), 2001.

[11] C.-Y. Chan, W. Fan, P. Felber, M. Garofalakis, and R. Rastogi. Tree pattern aggregation for scalable XML data dissemination. In $V L D B, 2002$.

[12] C.-Y. Chan, P. Felber, M. Garofalakis, and R. Rastogi. Efficient filtering of XML documents with XPath expressions. VLDB Journal, 11(4), 2002.

[13] R. Chand and P. A. Felber. A scalable protocol for content-based routing in overlay networks. In NCA, 2003.

[14] Y. Diao, M. Altinel, M. Franklin, H. Zhang, and P. Fischer. Path sharing and predicate evaluation for high-performance XML filtering. ACM TODS, 28(4), 2003.

[15] P. M. Fischer and D. Kossmann. Batched processing for information filters. In ICDE, 2005.

[16] X. Gong, W. Qian, Y. Yan, and A. Zhou. Bloom filter-based XML packets filtering for millions of path queries. In $I C D E$, 2005.

[17] T. J. Green, G. Miklau, M. Onizuka, and D.Suciu. Processing XML streams with deterministic automata. In ICDT, 2003.

[18] A. Gupta, A. Halevy, and D. Suciu. View selection for XML stream processing. In $W e b D B, 2002$.

[19] A. Gupta and D. Suciu. Stream processing of XPath queries with predicates. In SIGMOD, 2003.

[20] A. Gupta, D. Suciu, and A. Halevy. The view selection problem for XML content based routing. In PODS, 2003.

[21] S. Hou and H.-A. Jacobsen. Predicate-based filtering of XPath expressions. In ICDE, 2006.

[22] J. Kwon, P. Rao, B. Moon, and S. Lee. Fist: Scalable XML document filtering by sequencing twig patterns. In $V L D B$, 2005.

[23] D. S. Rosenblum and A. L. Wolf. A design framework for internet-scale event observation and notification. In ESEC/FSE-5, 1997.

[24] B. Segall, D. Arnold, J. Boot, M. Henderson, and T. Phelps. Content based routing with Elvin4. In AUUG2K, 2000.

[25] W3C. XML path language (XPath). 1999. http://www.w3.org/TR/xpath.

[26] X. Zhang, L. H. Yang, M. L. Lee, and W. Hsu. Scaling SDI systems via query clustring and aggregation. In $D A S F A A$, 2004. 